Research Paper

\title{
Burnout, Job Satisfaction, and Medical Malpractice among Physicians
}

Kuan-Yu Chen"1,2, , Che-Ming Yang4,5, Che-Hui Lien6, Hung-Yi Chiou1, Mau-Roung Lin², Hui-Ru Chang7, Wen-Ta Chiu $1,2,3, \bowtie$

1. School of Public Health, Taipei Medical University, Taiwan;

2. Graduate Institute of Injury Prevention and Control, Taipei Medical University, Taiwan;

3. Department of Health, Taiwan;

4. School of Health Care Administration, Taipei Medical University, Taiwan;

5. Taipei Medical University - Shuang Ho Hospital, Taiwan;

6. School of Law, Chung Yuan Christian University, Taiwan;

7. Long-term Care Insurance Preparatory Task Force, Department of Health, Taiwan.

$\bowtie$ Corresponding author: Wen-Ta Chiu, Chief, MD, PhD. Department of Health. E-mail: d508097004@tmu.edu.tw.

() Ivyspring International Publisher. This is an open-access article distributed under the terms of the Creative Commons License (http://creativecommons.org/ licenses/by-nc-nd/3.0/). Reproduction is permitted for personal, noncommercial use, provided that the article is in whole, unmodified, and properly cited.

Received: 2013.05.22; Accepted: 2013.08.23; Published: 2013.08.28

\begin{abstract}
Objectives: Our objective was to estimate the incidence of recent burnout in a large sample of Taiwanese physicians and analyze associations with job related satisfaction and medical malpractice experience.

Methods: We performed a cross-sectional survey. Physicians were asked to fill out a questionnaire that included demographic information, practice characteristics, burnout, medical malpractice experience, job satisfaction, and medical error experience. There are about $2 \%$ of total physicians. Physicians who were members of the Taiwan Society of Emergency Medicine, Taiwan Surgical Association, Taiwan Association of Obstetrics and Gynecology, The Taiwan Pediatric Association, and Taiwan Stroke Association, and physicians of two medical centers, three metropolitan hospitals, and two local community hospitals were recruited.

Results: There is high incidence of burnout among Taiwan physicians. In our research, Visiting staff (VS) and residents were more likely to have higher level of burnout of the emotional exhaustion (EE) and depersonalization (DP), and personal accomplishment (PA). There was no difference in burnout types in gender. Married had higher-level burnout in EE. Physicians who were 20 30 years old had higher burnout levels in EE, those $31 \sim 40$ years old had higher burnout levels in DP, and PA. Physicians who worked in medical centers had a higher rate in EE, DP, and who worked in metropolitan had higher burnout in PA. With specialty-in-training, physicians had higher-level burnout in EE and DP, but lower burnout in PA. Physicians who worked 13-17hr continuously had higher-level burnout in EE. Those with $\geq 41$ times/week of being on call had higher-level burnout in EE and DP. Physicians who had medical malpractice experience had higher-level burnout in EE, DP, and PA. Physicians who were not satisfied with physician-patient relationships had higher-level burnout than those who were satisfied.

Conclusion: Physicians in Taiwan face both burnout and a high risk in medical malpractice. There is high incidence of burnout among Taiwan physicians. This can cause shortages in medical care human resources and affect patient safety. We believe that high burnout in physicians was due to long working hours and several other factors, like mental depression, the evaluation assessment system, hospital culture, patient-physician relationships, and the environment. This is a very important issue on public health that Taiwanese authorities need to deal with.
\end{abstract}

Key words: Physician burnout, Medical malpractice, Job Satisfaction, Duty hour limitation. 


\section{Introduction}

Physicians take care of patients, but they often ignore their own health. Physician burnout is highly associated with medical errors, quality of care [1-3], and career satisfaction [ [ 2628 29] about one's career. One study found that, surgeons with work-home conflicts were more likely to have burnout, depression and less likely to recommend surgery as a career option to their children [29]. Burnout not only affects patient safety but more importantly physicians' health. Burnout is more prevalent among males, residents, and surgeons [4-9]. Previous studies focused on gender [10], age, medical errors [11 12], sleep deprivation, residents [4], the number of times on call per week [13], and specialties [26 28], but sometimes different results were obtained by different studies.

The 1999 Institute of Medicine's report estimated that medical errors in US hospitals result in at least 44,000 and as many as 98,000 deaths each year. Although there are different definitions of "medical error", a common definition is: a commission or omission with potentially negative consequences for the patient that would have been judged wrong by skilled and knowledgeable peers at the time it occurred independent of whether there were any negative consequences[14]. Several studies indicated that higher medical error rates are strongly associated with a physician's burnout status [11 15]. Furthermore, one study showed that after highly intensive work (80 $\mathrm{h}$ /week), physicians act like those with a blood alcohol concentration of $0.05 \%$ [13].

According to the news of Japan [16], 40\% of physicians face karoshi (a Japanese word meaning death from overwork), and nearly $80 \%$ produce medical error caused by burnout and sleep deprivation. Recent research by a labor policy research institution in Japan found that Japanese physicians work 53.2 $\mathrm{h}$ /week on average, $40 \%$ work up to $60 \mathrm{~h}$, and even $10 \%$ work over $80 \mathrm{~h}$. In our study $49.5 \%$ Taiwanese physicians work $>57 \mathrm{~h} /$ week, $34.5 \%$ work up to $65 \mathrm{~h}$ / week; Extra work hours for morning meeting, academic research, and teaching take $10.6 \%$ physicians $21 \mathrm{~h}$ in average. Furthermore, $83.9 \%$ of physicians admit that they nearly were negligent due to their burnout status, and nearly $85 \%$ produced adverse medical events. According to The Labor Standards Act of Japan, the total number of working hours should not exceed $40 \mathrm{~h} /$ week. Working overtime up to 80 $\mathrm{h} /$ month for 6 months would raise the risk of karoshi. There were 7 physicians who died of karoshi (a Japanese word for "death from overwork") in Taiwan from 2008 to 2012. However, little is known about the personal characteristics, work-related characteristics, and medical malpractice experience with burnout types among Taiwanese physicians. This is the first study on burnout of Taiwanese physicians.

\section{Material and Methods}

\section{Participants}

We conducted a survey to evaluate burnout and medical errors among Taiwanese physicians in May to November 2012. Physicians who were members of the Taiwan Society of Emergency Medicine, Taiwan Surgical Association, Taiwan Association of Obstetrics and Gynecology, The Taiwan Pediatric Association, and Taiwan Stroke Association, and physicians of two medical centers, three metropolitan hospitals, and two local community hospitals were recruited.

\section{Data collection}

We set purposive sampling to collect our samples. Physicians were surveyed in May to November 2012 with a three-part high reliable level [17] questionnaire $(\alpha=0.659)$ of 69 questions, which included demographic information, practice characteristics, burnout, medical malpractice experience, job satisfaction, and medical error experience. A cover letter stated the purpose of the survey was to better understand participants. Standardized tools were used for the burnout survey, the Maslach Burnout Inventory-General Survey [18], which has 3 subscales to evaluate the 3 domains of burnout: emotional exhaustion (EE), depersonalization (DP), and low personal accomplishment (PA). EE estimates feelings of being emotionally overextended and exhausted by one's work; DP estimates an unfeeling and impersonal response toward recipients of one's service, care treatment, or instruction; and PA estimates feelings of competence and successful achievement in one's work. Each question was answered on a 7-point Likert scale with response options ranging from "never" to "daily." Surgeons with a high score for medical professionals on the DP and/or EE subscales were considered to have at least one manifestation of professional burnout. The visitor was trained before study. The standard protocol was used to collect questionnaire and input data. There were totally sent out 1,100 questionnaires, 839 were returned, and excluded 30 missing. There are totally 809 into our analysis.

\section{Statistical analysis}

Descriptive statistics were used to characterize the sample demographics. Of the approximately 1,100 questionnaires we sent out, 839 (76\%) were returned. A Chi-squared test was used to estimate associations between the variables and burnout. Multivariate associations among demographic characteristics, professional characteristics, and medical malpractice ex- 
perience with burnout were assessed using a logistic regression. Both forward and backward elimination methods were used to select significant variables for the models where the directionality of the modeling did not impact the results. The independent variables used in these models included: alcohol use, average work hours per week, total work hours per week, number of times on call per week, medical malpractice experience, satisfaction with one's practice specialty, satisfaction with patient-physician relationships, and burnout. In addition, the odds ratio (OR) for reporting burnout associated with independent variables was also calculated. All analyses were done using SPSS vers. 19.0 (SPSS, Chicago, IL, USA).

\section{Results}

Physicians who were members of Taiwan Society of Emergency Medicine, Taiwan Surgical Association, Taiwan Association of Obstetrics and Gynecology, The Taiwan Pediatric Association, and Taiwan Stroke Association, and physicians of two medical centers, three metropolitan hospitals, and two local community hospitals were recruited. Approximately 839 physicians (about $2 \%$ of total physicians in the country) participated and returned the questionnaire, for a response rate of $76 \%$. The demographic and practice characteristics of the study participants are summarized in Table 1 . There were $36.8 \%$ who reported a moderate level of burnout, and $13.1 \%$ with a high level of burnout in EE. For DP, 32.5\% participant reported a moderate level of burnout, and 9.3\% reported a high level of burnout. For the PA type, $49.9 \%$, $49.3 \%$, and $0.7 \%$ respectively had high, moderate, and low levels of burnout. Approximately $65.5 \%$ of the study participants were married, $65.6 \%$ were Visiting staff (VS), and $79.4 \%$ were male. Based on official data from the Taiwan Medical Association (TMA) on demographics of Taiwanese members in 2011, 84\% of all TMA members were male, and the proportional distributions of physicians in each region of the country were also similar to TMA. The proportions of participants' service types were academic medical centers $(48.9 \%)$, metropolitan hospitals $(19.7 \%)$, local community hospitals $(8.7 \%)$, and physician clinics $(22.7 \%)$. Of the total, $360(44.5 \%)$ physicians had practiced $\geq 7$ years. Specialties of participants included medical department (19.0\%), surgery department $(11.2 \%)$, obstetrics-gynecology $(11.1 \%)$, pediatrics $(13.5 \%)$, emergency medicine (10.6\%), neurosurgery (7.5\%), dermatology $(2.1 \%)$, anesthesiology $(2.3 \%)$, ophthalmology $(2.0 \%)$, orthopedics $(3.1 \%)$, family medicine $(5.1 \%)$, those who had not passed a specialty test $(8.2 \%)$, and others $(4.2 \%)$. There were $12.7 \%$ of physicians who serviced $\geq 51$ patients each shift, and $33.4 \%$ worked continuously for $>13 \mathrm{~h}$ each shift. Collective- ly, 457 (56.5\%) physicians reportedly had medical malpractice experience, $80(10 \%)$ had up to 6 instances of medical malpractice experience within the 3 years before the survey, and $129(15.9 \%)$ had experience with malpractice lawsuits. There were $62.3 \%$ of physicians who were unsatisfied with physician-patient relationships, and $29.5 \%$ were unsatisfied with their practice specialty. The incidence rate of burnout types was shown in Table 2 .There are 26 physicians $/ 10,000$ at high risk in EE, 19 physicians $/ 10,000$ at high risk in $\mathrm{DP}$, and 101 physicians/10,000 at high risk in PA. We performed a Chi-squared test to identify factors associated with EE, DP, and PA. Relationships of demographics, practice characteristics, and malpractice experience with professional characteristics are shown in Table 3. Physician category, practice specialty, continuous hours works, total work hours per week, numbers of medical errors, age, hospital type, relationship status, years in practice, the number of patients serviced per shift, the number of times on call per week, medical malpractice experience, satisfaction with patient relationships, and satisfaction with the practice specialty were significant to EE. Hospital type, physician category, alcohol use, practice specialty, the number of patients serviced per shift, continuous hours works, total work hours per week, the number of times on call per week. Age, relationship status, years in practice, satisfaction with the practice specialty, and satisfaction with patient relationships were strongly associated with DP. Medical malpractice experience, years in practice, numbers of patients serviced per shift, number of times on call per week, physician category, gender, age, relationship status, practice specialty, satisfaction with practice specialty, and satisfaction with patient relationships were strongly association with PA (almost $\mathrm{p}=0.000$ ).

After controlling for other factors in the multiple-regression model analysis, physicians who used alcohol compared to those who did not had a 2.8-fold risk of DP ( $p=0.004)$, after working $\geq 18$ continuous hours had a 14.7-fold risk of low-level burnout and a 2.8-fold risk of moderate-level burnout in EE compared to those with $\geq 3 \sim 7$ continuous working hours ( $p=0.005$ and 0.268 ). Physicians who worked $\geq 65$ $\mathrm{h} /$ week had a 1.4-fold risk of low-level burnout compared to those who worked 49 56 h/week, and a 1.5 -fold risk of moderate-level burnout in EE ( $p=0.02$ and 0.009). Those with the number of times on call per week of $\geq 41$, had a 2.4 -fold risk of low-level burnout in EE compared to physicians who did not go on call $(p=0.028)$. Those who went on call $\geq 41$ times also had a higher risk in PA $(p=0.014)$. Physicians who had medical malpractice experience had a higher risk of high-level burnout in PA than those with no experience $(p=0.098)$. Physicians who were not satisfied with 
their specialty, who had not chosen a specialty, and who were worried about the future had higher risks of high-level burnout in DP and PA. Those who were extremely dissatisfied with patient-physicians rela- tionships had a 22.1-fold risk of low-level burnout in EE $(p=0.004)$, and had a higher risk of high-level burnout in DP than who were satisfied $(p=0.000)$ (Table 4).

Table I. Personal and Practice Characteristics $N=(809)$.

\begin{tabular}{|c|c|}
\hline \multicolumn{2}{|l|}{ Personal characteristics } \\
\hline \multicolumn{2}{|l|}{ Age (years) } \\
\hline Median 31 40 & $n(\%)$ \\
\hline $20 \sim 30$ & $162(20.0)$ \\
\hline $31 \sim 40$ & $307(37.9)$ \\
\hline $41 \sim 50$ & $182(22.5)$ \\
\hline$\geq 51$ & $158(19.5)$ \\
\hline \multicolumn{2}{|l|}{ Gender } \\
\hline Male & $642(79.4)$ \\
\hline Female & $167(20.6)$ \\
\hline \multicolumn{2}{|l|}{ Relationship status } \\
\hline Single & $267(33.0)$ \\
\hline Married & $530(65.5)$ \\
\hline Others & $12(1.5)$ \\
\hline \multicolumn{2}{|l|}{ Consume alcohol } \\
\hline Yes & $110(13.6)$ \\
\hline No & $699(86.4)$ \\
\hline \multicolumn{2}{|l|}{ Practice characteristics } \\
\hline \multicolumn{2}{|l|}{ Physician category } \\
\hline Intern & $54(6.7)$ \\
\hline Resident & $224(27.7)$ \\
\hline Visiting staff (VS) & $531(65.6)$ \\
\hline \multicolumn{2}{|l|}{ Hospital type } \\
\hline Academic medical center & $396(48.9)$ \\
\hline Metropolitan hospital & $159(19.7)$ \\
\hline Local community hospital & $70(8.7)$ \\
\hline Physician clinic & $184(22.7)$ \\
\hline \multicolumn{2}{|l|}{ Years in practice (years) } \\
\hline $1 \sim 3$ & $147(18.2)$ \\
\hline $4 \sim 6$ & $117(14.5)$ \\
\hline $7 \sim 9$ & $79(9.8)$ \\
\hline$\geq 10$ & $281(34.7)$ \\
\hline Specialist-in-training & $185(22.9)$ \\
\hline \multicolumn{2}{|l|}{ Practice specialty } \\
\hline Medical department & $154(19.0)$ \\
\hline Surgery department & $91(11.2)$ \\
\hline Obstetrics-gynecology & $90(11.1)$ \\
\hline Pediatrics & $109(13.5)$ \\
\hline Emergency medicine & $86(10.6)$ \\
\hline Neurological surgery & $61(7.5)$ \\
\hline Dermatology & $17(2.1)$ \\
\hline Anesthesiology & $19(2.3)$ \\
\hline Ophthalmology & $16(2.0)$ \\
\hline Orthopedics & $25(3.1)$ \\
\hline Specialist-in-training & $66(8.2)$ \\
\hline Family medicine & $41(5.1)$ \\
\hline Others & $34(4.2)$ \\
\hline \multicolumn{2}{|c|}{ Number of patients serviced per shift (average) } \\
\hline $10 \sim 50$ & $518(64.0)$ \\
\hline$\geq 51$ & $103(12.7)$ \\
\hline Specialist-in-training & $188(23.2)$ \\
\hline \multicolumn{2}{|l|}{ Hours worked per shift (h) } \\
\hline $3 \sim 7$ & $104(12.9)$ \\
\hline $8 \sim 12$ & $434(53.6)$ \\
\hline $13 \sim 17$ & $235(29.0)$ \\
\hline
\end{tabular}




\begin{tabular}{ll}
\hline$\geq 18$ & $36(4.4)$ \\
Total work hours per week $(\mathbf{h})$ & $74(9.1)$ \\
$>39$ & $169(20.9)$ \\
$40 \sim 48$ & $166(20.5)$ \\
$49 \sim 56$ & $121(15.0)$ \\
$57 \sim 64$ & $279(34.5)$ \\
$\geq 65$ & $214(26.5)$ \\
Number of times on call per week (average) & $237(29.3)$ \\
None & $119(14.7)$ \\
$1 \sim 20$ & $64(7.9)$ \\
$21 \sim 30$ & $175(21.6)$ \\
$31 \sim 40$ & \\
$\geq 41$ & $457(56.5)$ \\
Medical malpractice experience & $352(43.5)$ \\
Yes & \\
No &
\end{tabular}

Table 2. Incidence Rate of Burnout Types among Taiwanese Physicians (per 10,000 physicians).

\begin{tabular}{lllll}
\hline & EE & DP & PA \\
\hline Low level & 100.8 & 117.2 & 1.5 \\
Moderate level & 74.2 & 65.5 & 99.3 \\
High level & 26.4 & 18.7 & 100.5 \\
\hline
\end{tabular}

Table 3. Relation between Burnout, Personal and Practice Characteristics

\begin{tabular}{|c|c|c|c|c|c|c|c|c|c|c|c|c|c|}
\hline & & \multicolumn{3}{|l|}{ EE } & \multirow[t]{2}{*}{$\mathbf{P}$} & \multicolumn{3}{|l|}{ DP } & \multirow[t]{2}{*}{$\mathbf{P}$} & \multicolumn{3}{|l|}{ PA } & \multirow[t]{2}{*}{$\mathbf{P}$} \\
\hline & & $\begin{array}{l}\text { Low } \\
\text { level } \\
\text { burnout }\end{array}$ & $\begin{array}{l}\text { Moderate } \\
\text { level } \\
\text { burnout }\end{array}$ & $\begin{array}{l}\text { High } \\
\text { level } \\
\text { burnout }\end{array}$ & & $\begin{array}{l}\text { Low } \\
\text { level } \\
\text { burnout }\end{array}$ & $\begin{array}{l}\text { Moderate } \\
\text { level } \\
\text { burnout }\end{array}$ & $\begin{array}{l}\text { High } \\
\text { level } \\
\text { burnout }\end{array}$ & & $\begin{array}{l}\text { High } \\
\text { level } \\
\text { burnout }\end{array}$ & $\begin{array}{l}\text { Moderate } \\
\text { level } \\
\text { burnout }\end{array}$ & $\begin{array}{l}\text { Low } \\
\text { level } \\
\text { burnout }\end{array}$ & \\
\hline \multirow[t]{4}{*}{ Age } & $20-30 y$ & $17.0 \%$ & $19.1 \%$ & $34.0 \%$ & \multirow[t]{4}{*}{0.000} & $16.0 \%$ & $23.6 \%$ & $33.3 \%$ & \multirow[t]{4}{*}{0.000} & $14.1 \%$ & $25.1 \%$ & $83.3 \%$ & \multirow[t]{4}{*}{0.000} \\
\hline & $31-40 y$ & $35.3 \%$ & $42.3 \%$ & $35.8 \%$ & & $37.4 \%$ & $38.8 \%$ & $37.3 \%$ & & $38.6 \%$ & $37.6 \%$ & $16.7 \%$ & \\
\hline & $41-50 y$ & $21.7 \%$ & $22.8 \%$ & $24.5 \%$ & & $21.9 \%$ & $24.0 \%$ & $21.3 \%$ & & $27.0 \%$ & $18.3 \%$ & $0.0 \%$ & \\
\hline & $\geqq 51 \mathrm{y}$ & $25.9 \%$ & $15.8 \%$ & $5.7 \%$ & & $24.7 \%$ & $13.7 \%$ & $8.0 \%$ & & $20.3 \%$ & $19.0 \%$ & $0.0 \%$ & \\
\hline \multirow[t]{3}{*}{ Physician category } & Intern & $5.9 \%$ & $6.7 \%$ & $9.4 \%$ & \multirow[t]{3}{*}{0.002} & $6.0 \%$ & $8.0 \%$ & $6.7 \%$ & \multirow[t]{3}{*}{0.009} & $5.2 \%$ & $8.0 \%$ & $16.7 \%$ & \multirow[t]{3}{*}{0.000} \\
\hline & Resident & $23.7 \%$ & $28.2 \%$ & $41.5 \%$ & & $23.8 \%$ & $30.4 \%$ & $41.3 \%$ & & $21.5 \%$ & $33.3 \%$ & $66.7 \%$ & \\
\hline & v.s. & $70.4 \%$ & $65.1 \%$ & $49.1 \%$ & & $70.2 \%$ & $61.6 \%$ & $52.0 \%$ & & $73.3 \%$ & $58.6 \%$ & $16.7 \%$ & \\
\hline \multirow{3}{*}{$\begin{array}{l}\text { Relationship } \\
\text { status }\end{array}$} & Single & $29.9 \%$ & $33.9 \%$ & $42.5 \%$ & \multirow[t]{3}{*}{0.055} & $29.1 \%$ & $36.5 \%$ & $45.3 \%$ & \multirow[t]{3}{*}{0.028} & $27.7 \%$ & $37.8 \%$ & $66.7 \%$ & \multirow[t]{3}{*}{0.006} \\
\hline & Married & $68.9 \%$ & $63.8 \%$ & $57.5 \%$ & & $68.9 \%$ & $62.7 \%$ & $53.3 \%$ & & $71.3 \%$ & $60.2 \%$ & $33.3 \%$ & \\
\hline & Others & $1.2 \%$ & $2.3 \%$ & $0.0 \%$ & & $1.9 \%$ & $0.8 \%$ & $1.3 \%$ & & $1.0 \%$ & $2.0 \%$ & $0.0 \%$ & \\
\hline \multirow[t]{5}{*}{ Years in practice } & $1-3 y$ & $20.2 \%$ & $17.4 \%$ & $12.3 \%$ & \multirow[t]{5}{*}{0.000} & $18.9 \%$ & $16.7 \%$ & $18.7 \%$ & \multirow[t]{5}{*}{0.019} & $19.3 \%$ & $17.3 \%$ & $0.0 \%$ & \multirow[t]{5}{*}{0.000} \\
\hline & $4-6 y$ & $12.3 \%$ & $15.1 \%$ & $20.8 \%$ & & $13.2 \%$ & $16.3 \%$ & $16.0 \%$ & & $13.4 \%$ & $15.8 \%$ & $0.0 \%$ & \\
\hline & $7-9 y$ & $8.1 \%$ & $12.4 \%$ & $8.5 \%$ & & $9.1 \%$ & $10.6 \%$ & $10.7 \%$ & & $9.2 \%$ & $10.3 \%$ & $16.7 \%$ & \\
\hline & $\geqq 10 \mathrm{y}$ & $40.2 \%$ & $32.6 \%$ & $19.8 \%$ & & $39.8 \%$ & $28.9 \%$ & $24.0 \%$ & & $41.1 \%$ & $28.8 \%$ & $0.0 \%$ & \\
\hline & Specialist-in-training & $19.0 \%$ & $22.5 \%$ & $38.7 \%$ & & $18.9 \%$ & $27.4 \%$ & $30.7 \%$ & & $17.1 \%$ & $27.8 \%$ & $83.3 \%$ & \\
\hline \multirow{3}{*}{$\begin{array}{l}\text { Service patient } \\
\text { numbers per time }\end{array}$} & $10-50 \mathrm{P}$ & $67.9 \%$ & $62.8 \%$ & $52.8 \%$ & \multirow[t]{3}{*}{0.001} & $66.6 \%$ & $62.4 \%$ & $54.7 \%$ & 0.004 & $67.6 \%$ & $61.4 \%$ & $0.0 \%$ & 0.000 \\
\hline & $\geqq 51 \mathrm{P}$ & $13.6 \%$ & $13.1 \%$ & $8.5 \%$ & & $14.7 \%$ & $9.5 \%$ & $12.0 \%$ & & $14.6 \%$ & $10.8 \%$ & $16.7 \%$ & \\
\hline & Specialist-in-training & $18.5 \%$ & $24.2 \%$ & $38.7 \%$ & & $18.7 \%$ & $28.1 \%$ & $33.3 \%$ & & $17.8 \%$ & $27.8 \%$ & $83.3 \%$ & \\
\hline Continuous work & $3 \mathrm{hr}-7 \mathrm{hr}$ & $19.8 \%$ & $7.4 \%$ & $1.9 \%$ & 0.000 & $17.7 \%$ & $6.8 \%$ & $4.0 \%$ & 0.000 & $14.1 \%$ & $11.8 \%$ & $0.0 \%$ & 0.642 \\
\hline hours/ time & $8 \mathrm{hr}-12 \mathrm{hr}$ & $57.0 \%$ & $53.4 \%$ & $41.5 \%$ & & $55.7 \%$ & $52.5 \%$ & $45.3 \%$ & & $54.0 \%$ & $53.4 \%$ & $50.0 \%$ & \\
\hline & 13hr-17hr & $21.2 \%$ & $33.9 \%$ & $45.3 \%$ & & $23.8 \%$ & $35.7 \%$ & $37.3 \%$ & & $27.0 \%$ & $30.8 \%$ & $50.0 \%$ & \\
\hline & $\geqq 18 \mathrm{hr}$ & $2.0 \%$ & $5.4 \%$ & $11.3 \%$ & & $2.8 \%$ & $4.9 \%$ & $13.3 \%$ & & $5.0 \%$ & $4.0 \%$ & $0.0 \%$ & \\
\hline Total work hours / & $>39 \mathrm{hr}$ & $13.1 \%$ & $7.0 \%$ & $0.0 \%$ & 0.000 & $11.7 \%$ & $6.8 \%$ & $1.3 \%$ & 0.014 & $9.4 \%$ & $8.8 \%$ & $16.7 \%$ & 0.777 \\
\hline week & $40 \mathrm{hr}-48 \mathrm{hr}$ & $24.4 \%$ & $19.1 \%$ & $12.3 \%$ & & $22.1 \%$ & $20.2 \%$ & $16.0 \%$ & & $18.8 \%$ & $23.1 \%$ & $16.7 \%$ & \\
\hline & $49 \mathrm{hr}-56 \mathrm{hr}$ & $22.7 \%$ & $19.8 \%$ & $14.2 \%$ & & $21.1 \%$ & $20.9 \%$ & $16.0 \%$ & & $23.0 \%$ & $18.0 \%$ & $16.7 \%$ & \\
\hline & $57 \mathrm{hr}-64 \mathrm{hr}$ & $11.6 \%$ & $17.4 \%$ & $20.8 \%$ & & $13.0 \%$ & $16.7 \%$ & $21.3 \%$ & & $14.6 \%$ & $15.3 \%$ & $16.7 \%$ & \\
\hline & $\geqq 65 \mathrm{hr}$ & $28.1 \%$ & $36.6 \%$ & $52.8 \%$ & & $32.1 \%$ & $35.4 \%$ & $45.3 \%$ & & $34.2 \%$ & $34.8 \%$ & $33.3 \%$ & \\
\hline On call numbers / & None & $33.6 \%$ & $20.8 \%$ & $15.1 \%$ & 0.000 & $31.9 \%$ & $19.0 \%$ & $18.7 \%$ & 0.000 & $26.5 \%$ & $26.8 \%$ & $0.0 \%$ & 0.029 \\
\hline week & $1-20$ & $30.9 \%$ & $29.2 \%$ & $23.6 \%$ & & $28.9 \%$ & $32.7 \%$ & $20.0 \%$ & & $27.2 \%$ & $31.3 \%$ & $33.3 \%$ & \\
\hline & $21-30$ & $14.8 \%$ & $15.1 \%$ & $13.2 \%$ & & $12.3 \%$ & $19.4 \%$ & $13.3 \%$ & & $12.9 \%$ & $16.5 \%$ & $16.7 \%$ & \\
\hline & $31-40$ & $6.9 \%$ & $9.1 \%$ & $8.5 \%$ & & $7.2 \%$ & $8.7 \%$ & $9.3 \%$ & & $6.7 \%$ & $9.0 \%$ & $16.7 \%$ & \\
\hline & $\geqq 41$ & $13.8 \%$ & $25.8 \%$ & $39.6 \%$ & & $19.6 \%$ & $20.2 \%$ & $38.7 \%$ & & $26.7 \%$ & $16.3 \%$ & $33.3 \%$ & \\
\hline Medical malprac- & Yes & $51.6 \%$ & $63.4 \%$ & $55.7 \%$ & 0.007 & $56.2 \%$ & $55.9 \%$ & $61.3 \%$ & 0.680 & $59.2 \%$ & $54.6 \%$ & $0.0 \%$ & 0.009 \\
\hline tice experience & No & $48.4 \%$ & $36.6 \%$ & $44.3 \%$ & & $43.8 \%$ & $44.1 \%$ & $38.7 \%$ & & $40.8 \%$ & $45.4 \%$ & $100.0 \%$ & \\
\hline Satisfied with & Yes & $68.9 \%$ & $52.7 \%$ & $32.1 \%$ & 0.000 & $68.1 \%$ & $50.6 \%$ & $22.7 \%$ & 0.000 & $63.6 \%$ & $53.1 \%$ & $16.7 \%$ & 0.000 \\
\hline
\end{tabular}




\begin{tabular}{|c|c|c|c|c|c|c|c|c|c|c|c|c|c|}
\hline \multirow{4}{*}{ practice specialty } & & \multicolumn{3}{|l|}{ EE } & \multirow[t]{2}{*}{$\mathbf{P}$} & \multicolumn{3}{|l|}{ DP } & \multirow[t]{2}{*}{$\mathbf{P}$} & \multicolumn{3}{|l|}{ PA } & \multirow[t]{2}{*}{$\mathbf{P}$} \\
\hline & & $\begin{array}{l}\text { Low } \\
\text { level } \\
\text { burnout }\end{array}$ & $\begin{array}{l}\text { Moderate } \\
\text { level } \\
\text { burnout }\end{array}$ & $\begin{array}{l}\text { High } \\
\text { level } \\
\text { burnout }\end{array}$ & & $\begin{array}{l}\text { Low } \\
\text { level } \\
\text { burnout }\end{array}$ & $\begin{array}{l}\text { Moderate } \\
\text { level } \\
\text { burnout }\end{array}$ & $\begin{array}{l}\text { High } \\
\text { level } \\
\text { burnout }\end{array}$ & & $\begin{array}{l}\text { High } \\
\text { level } \\
\text { burnout }\end{array}$ & $\begin{array}{l}\text { Moderate } \\
\text { level } \\
\text { burnout }\end{array}$ & $\begin{array}{l}\text { Low } \\
\text { level } \\
\text { burnout }\end{array}$ & \\
\hline & No & $21.5 \%$ & $34.6 \%$ & $46.2 \%$ & & $23.4 \%$ & $34.2 \%$ & $52.0 \%$ & & $29.0 \%$ & $29.8 \%$ & $50.0 \%$ & \\
\hline & $\begin{array}{l}\text { Specialist-in-training } \\
\text { but unsatisfy }\end{array}$ & $7.2 \%$ & $10.7 \%$ & $19.8 \%$ & & $6.0 \%$ & $13.3 \%$ & $25.3 \%$ & & $5.9 \%$ & $14.3 \%$ & $16.7 \%$ & \\
\hline \multirow{6}{*}{$\begin{array}{l}\text { Satisfied with } \\
\text { patient-physician } \\
\text { relationship }\end{array}$} & $\begin{array}{l}\text { Specialist-in-training } \\
\text { but satisfy }\end{array}$ & $2.5 \%$ & $2.0 \%$ & $1.9 \%$ & & $2.6 \%$ & $1.9 \%$ & $0.0 \%$ & & $1.5 \%$ & $2.8 \%$ & $16.7 \%$ & \\
\hline & Extremely satisfy & $1.2 \%$ & $0.7 \%$ & $0.0 \%$ & \multirow[t]{5}{*}{0.000} & $1.5 \%$ & $0.0 \%$ & $0.0 \%$ & \multirow[t]{5}{*}{0.000} & $0.7 \%$ & $0.8 \%$ & $16.7 \%$ & \multirow[t]{5}{*}{0.000} \\
\hline & Satisfy & $10.1 \%$ & $4.7 \%$ & $0.9 \%$ & & $10.2 \%$ & $3.0 \%$ & $0.0 \%$ & & $8.7 \%$ & $5.3 \%$ & $0.0 \%$ & \\
\hline & Moderate & $42.5 \%$ & $19.8 \%$ & $10.4 \%$ & & $33.0 \%$ & $31.2 \%$ & $6.7 \%$ & & $27.2 \%$ & $32.8 \%$ & $16.7 \%$ & \\
\hline & Unsatisfy & $34.1 \%$ & $48.3 \%$ & $32.1 \%$ & & $37.0 \%$ & $43.7 \%$ & $36.0 \%$ & & $38.1 \%$ & $40.6 \%$ & $0.0 \%$ & \\
\hline & Extremely unsatisfy & $12.1 \%$ & $26.5 \%$ & $56.6 \%$ & & $18.3 \%$ & $22.1 \%$ & $57.3 \%$ & & $25.2 \%$ & $20.6 \%$ & $66.7 \%$ & \\
\hline
\end{tabular}

Table 4. Factors Independently Associated with Practiced Burnout Types on Multiple Regression Model

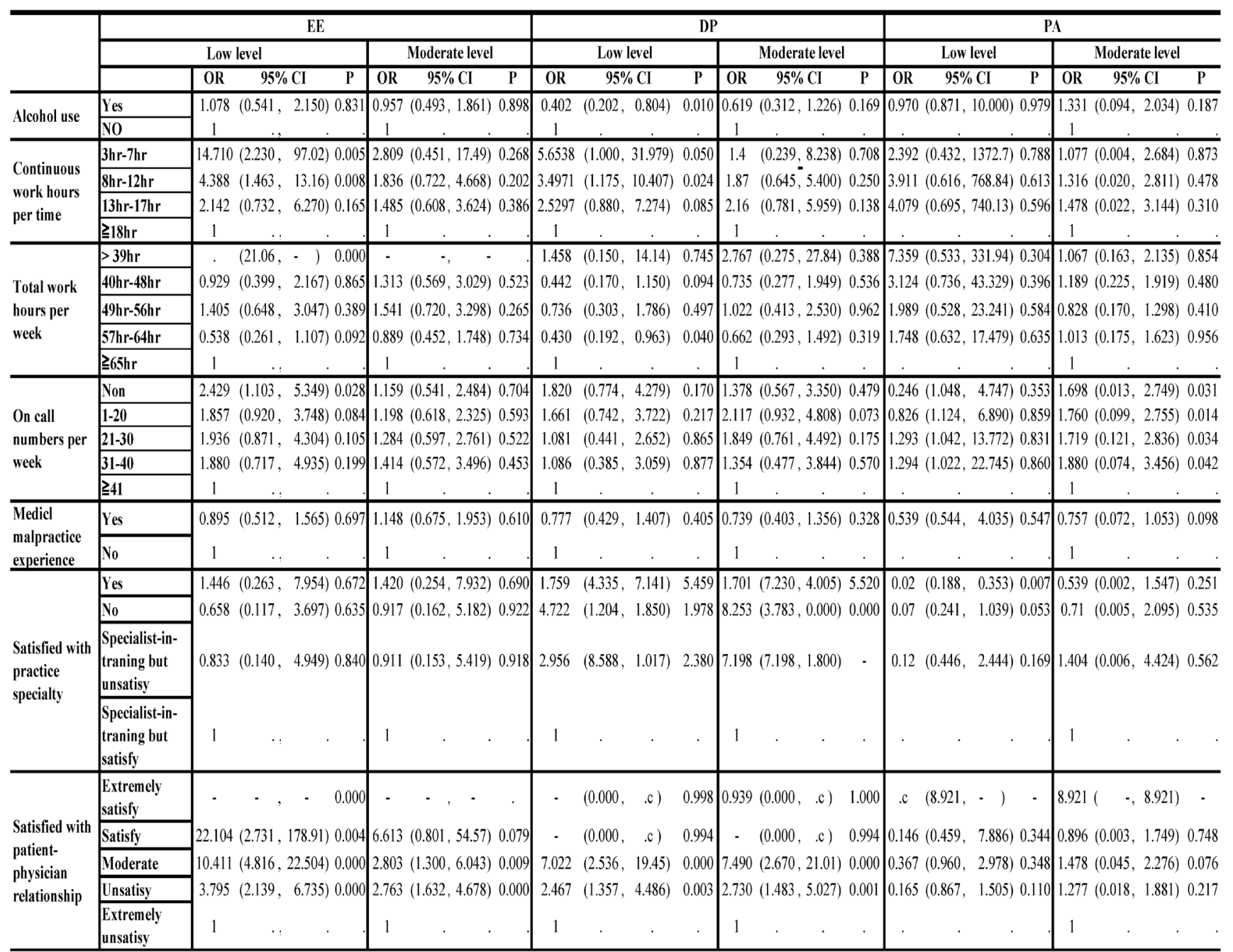

\section{Discussion}

In this article, burnout and medical malpractice experience were examined in a large sample of 809 physicians in Taiwan. Previous studies usually focused on gender [13], age, medical errors [11 12], sleep deprivation [4], residents [4], number of times on call per week [13], and specialties [26 28], but different results were sometimes obtained in different studies.
For instance on gender, Fahrenkopf et al. found that there was no association between gender and burnout [19 20], but another study found that women had a higher risk of burnout [21]. This discrepancy might have been caused by differences in subjects, instruments, specialties, research methods, and countries. In our study, there was no significant difference in burnout by gender. With the Chi-squared test, physician category, age, and relationship statuses were 
strongly associated with burnout, but there was no association with burnout in the multiple-regression models. Some studies found a higher incidence of burnout among residents and surgeons [1 5-8], while we found a higher incidence of burnout of PA, EE and DP in VS. The recent study, found that surgeons reporting a major medical error in the previous 3 months were more likely to have alcohol abuse, and the point prevalence for alcohol abuse or dependency on female surgeons (25.6\%) were higher than male $(13.9 \%)[27]$. In our study we found that physicians who with higher level burnout had more alcohol use when feeling depressed due to the heavy workload, the male physicians were more likely to have alcohol use than Female. Errors were strongly associated with EE with high-level burnout. Our result was similar to those of previous studies [22 23]. In a bivariate analysis, we found that age, working for $\geq 8$ $\mathrm{h} /$ shift, serving $\geq 51$ patients per shift, being on call $\geq 41$ times per week, having medical malpractice experience, not being satisfied with one's specialty, not being satisfied with patient-physician relationships, and the number of medical error were strongly associated with high-level burnout in EE (all $p<0.001)$. EE indicates feelings of being emotionally overextended and exhausted by one's work. It might not be difficult to understand that exhaustion was caused by those factors. Physicians with long continuous work hours, more on call numbers, unsatisfied with specialty, younger age, and those unsatisfied with patient-physician relationships were strongly associated with DP (all $p<0.001)$. DP indicates an unfeeling or impersonal response toward recipients of one's service, care treatment, or instruction. It is easy to realize that when physicians have a heavy burden and fears of being sued, they might indicate a higher risk of DP. PA indicates feelings of competence and successful achievement in one's work. In our study, we found that age, physician category, years in practice, service patient number per time, not being satisfied with one's practice specialty, and not being satisfied with patient-physician relationships were factors strongly associated with PA (all $p<0.001$ ). Medical malpractice rates at home and abroad have obviously been increasing in recent years. Medical malpractice cases in 2008 were approximately four times those in 2004 in Taiwan. According to statistics from the TMA in 2011, there were 40,183 physicians in Taiwan. The population of Taiwan was $23,261,747$ based on the official statistics of June 2012. This means that on average, one physician needs to care for 579 people, which indicates a heavy workload for physicians in Taiwan. In consideration of work pressure and high litigation risk, increasing numbers of medical students are inclined to choose low-risk specialties [24]. Medical treatment has the characteristics of urgency, necessity, and high-precision, which are much likely to cause excessive pressure on and even burnout in physicians.

There are some limitations of our study. First, we used self-reported data to determine physicians' burnout, medical malpractice experience, and medical errors. It is unknown whether physicians who have experienced medical malpractice/medical errors were less likely to respond to, under-reported, more likely to respond to, or over-reported burnout, because the topic has a great effect on them. We used a well-known instrument to estimate the data, and it has been used in similar studies. In addition, our sample size provided burnout estimates for about $2 \%$ of the total physician population with $95 \%$ confidence. Second, as to restrictions of our research subjects, it is hard to establish a random study. Although we used purposive sampling, we were careful to include relevant medical associations to increase our representation, and reached a high response rate (74\%) to avoid self-selection bias.

\section{Conclusions}

In conclusion, there was high incidence burnout in residents, and VS physicians had high-level burnout which was associated with greater alcohol use when feeling depressed about the heavy workload. The number of errors was strongly associated with EE in high-level burnout. Working $\geq 8 \mathrm{~h} /$ shift, serving $\geq 51$ patient per shift, being on call $\geq 41$ times per week, having medical malpractice experience, not being satisfied with one's specialty, not being satisfied with patient-physician relationships, and the number of medical errors, physician category, practice specialty, were strongly associated with high-level burnout in EE. Hospital type, physician category, alcohol use, practice specialty, the number of patients serviced per shift, continuous hours works, total work hours per week, the number of times on call per week. Age, relationship status, years in practice, satisfaction with the practice specialty, and satisfaction with patient relationships were strongly associated with DP. Medical malpractice experience, years in practice, numbers of patients serviced per shift, number of times on call per week, physician category, gender, age, relationship status, practice specialty, satisfaction with practice specialty, and satisfaction with patient relationships were strongly association with PA There were 7 physicians who died of karoshi in Taiwan from 2008 to 2012.It is important to restrict working times from the point of view of patient safety and physicians' health. In several developed countries [25], shown in table 5, there are duty hour limitations for physicians. This is not yet a reality for Taiwanese physicians. Since physicians in Taiwan face both 
burnout and high risks of medical malpractice ${ }^{1}$, that would cause medical care human resource shortages, choose lower risk specialty to practice and affect patient safety. We believe that high burnout in physicians, due to long working hours and several other factors, like mental depression, the evaluation assessment system, hospital cultures, patient-physician relationships, and the environment, is a very important issue that Taiwanese authorities need to address.

Table 5. Duty Hour Limitations and Mean Working Hours for Physicians in Different Countries.

\begin{tabular}{|c|c|c|c|c|c|}
\hline & US & Japan & European Union & New Zealand & Taiwan \\
\hline Mean hours of physicians' work in research & $51 \mathrm{~h} /$ week & $70.6 \mathrm{~h} /$ week & $46.3 \mathrm{~h} /$ week & 50 70 h / week & $46 \sim 85 \mathrm{~h} /$ week \\
\hline Physician's duty hour limitations & $80 \mathrm{~h} /$ week & $40 \mathrm{~h} /$ week & $48 \mathrm{~h} /$ week & $72 \mathrm{~h} /$ week & NA \\
\hline
\end{tabular}

\section{Competing Interests}

The authors have declared that no competing interest exists.

\section{References}

1. Shanafelt TD, Bradley KA, Wipf JE, Back AL. Burnout and self-reported patient care in an internal medicine residency program. Annals of internal medicine 2002;136(5):358-67.

2. Linzer M, Manwell LB, Williams ES, et al. Working conditions in primary care: physician reactions and care quality. Annals of internal medicine 2009;151(1):28-36

3. Shirom A, Nirel N, Vinokur AD. Overload, autonomy, and burnout as predictors of physicians' quality of care. Journal of occupational health psychology 2006;11(4):328-42.

4. Shanafelt TD, Sloan JA, Habermann TM. The well-being of physicians. The American journal of medicine 2003;114(6):513-9.

5. Wallace JE, Lemaire JB, Ghali WA. Physician wellness: a missing quality indicator. Lancet 2009;374(9702):1714-21.

6. Campbell DAJr., Sonnad SS, Eckhauser FE, Campbell KK, Greenfield LJ. Burnout among American surgeons. Surgery 2001;130(4):696-702.

7. Thomas NK. Resident burnout. JAMA : the journal of the American Medical Association 2004;292(23):2880-9.

8. Prins JT, Gazendam-Donofrio SM, Tubben BJ, van der Heijden FM, van de Wiel HB, Hoekstra-Weebers JE. Burnout in medical residents: a review. Medical education 2007;41(8):788-800.

9. Balch CM, Freischlag JA, Shanafelt TD. Stress and burnout among surgeons: understanding and managing the syndrome and avoiding the adverse consequences. Archives of surgery 2009;144(4):371-6.

10. Dyrbye LN, Shanafelt TD, Balch CM, Satele D, Sloan J, Freischlag J. Relationship between work-home conflicts and burnout among American surgeons: a comparison by sex. Archives of surgery 2011;146(2):211-7.

11. Shanafelt TD, Balch CM, Bechamps G, et al. Burnout and medical errors among American surgeons. Annals of surgery 2010;251(6):995-1000.

12. Lauris C. Kaldjian EWJ, Barry J. Wu,Valerie L. Forman-Hoffman,Benjamin H. Levi,Gary E. Rosenthal. Reporting Medical Errors to Improve Patient Safety A Survey of Physicians in Teaching Hospitals. Archives of internal medicine 2008;168(1):40-46

13. Liselotte N.Dyrbye TDS, Charles M. Balch,Jeff Sloan,Julie Freischlag. Relationship Between Work-Home Conflicts and Burnout Among American Surgeons A Comparison by Sex. Archives of surgery 2011;146(2):211-17

14. A W Wu SF, S J McPhee, B Lo. Do house officers learn from their mistakes? BMJ open 2003;12:221-28.

15. West CP, Tan AD, Habermann TM, Sloan JA, Shanafelt TD. Association of resident fatigue and distress with perceived medical errors. JAMA : the journal of the American Medical Association 2009;302(12):1294-300.

16. [Internet] http://www.chinadaily.com.cn/hqgi/bmoz/2012-09-25/ content_7099701.html

17. Cuieford. Fundamental Statistics in Psychology and Education. 1965.

18. Schaufeli WBLMP, Maslach C. \& Jackson SE. Maslach Burnout Inventory-General Survey (MBI-GS). 1996;:19-26.
19. Fahrenkopf AM, Sectish TC, Barger LK, et al. Rates of medication errors among depressed and burnt out residents: prospective cohort study. Bmj 2008;336(7642):488-91.

20. JENS KLEIN KGF, KARL BLUM AND OLAF VON DEM KNESEBECK. Burnout and perceived quality of care among German clinicians in surgery. International Journal for Quality in Health Care 2010;22:525-30.

21. Kuerer HM, Eberlein TJ, Pollock RE, et al. Career satisfaction, practice patterns and burnout among surgical oncologists: report on the quality of life of members of the Society of Surgical Oncology. Annals of surgical oncology 2007;14(11):3043-53.

22. West CP, Huschka MM, Novotny PJ, et al. Association of perceived medical errors with resident distress and empathy: a prospective longitudinal study. JAMA : the journal of the American Medical Association 2006;296(9):1071-8

23. Balch CM, Oreskovich MR, Dyrbye LN, et al. Personal consequences of malpractice lawsuits on American surgeons. Journal of the American College of Surgeons 2011;213(5):657-67.

24. Chen KY, Yang CM, Tsai SH, Chiou HY, Lin MR, Chiu WT. Medical malpractice in Taiwan: injury types, compensation, and specialty risk. Academic emergency medicine 2012;19(5):598-600.

25. Prokhorov AV, Winickoff JP, Ahluwalia JS, et al. Youth tobacco use: a global perspective for child health care clinicians. Pediatrics 2006 Sep;118(3):e890-903

26. Charles MB, Tait DS, Jeffrey AS, et al. Distress and Career Satisfaction Among 14 Surgical Specialties,Comparing Academic and Private Practice Settings. Annals of Surgery 2011; 254(4):558-568.

27. Michael R. Oreskovich, MD; Krista L. Kaups, MD; Charles M. Balch, MD,et al. Prevalence of Alcohol Use Disorders Among American Surgeons. Arch Surg. 2012;147(2):168-174.

28. Tait DS, Sonja B, Litjen T, et al. Burnout and Satisfaction With Work-Life Balance Among US Physicians Relative to the General US Population. Arch Intern Med.2012; 172 (18): 1377-85.

29. Liselotte ND, Julie F, Krista LK, et al. Work-Home Conflicts Have a Substantial Impact on Career Decisions That Affect the Adequacy of the Surgical Workforce. ARCH SURG 2012; 147(10): 933-939. 